\title{
Pneumatosis Coli Associated with Pseudomembranous Colitis in a Patient following Colonic Surgery
}

\author{
Jennifer Bailey and Eldon A. Shaffer \\ Division of Gastroenterology, Faculty of Medicine, University of Calgary, Teaching Research \& Wellness Building, Room 6D48, \\ 3280 Hospital Dr NW, Calgary, AB, Canada T2N4N1
}

Correspondence should be addressed to Eldon A. Shaffer, shaffer@ucalgary.ca

Received 16 June 2010; Revised 6 October 2010; Accepted 8 October 2010

Academic Editor: Anthony W. Chow

Copyright (๑) 2010 J. Bailey and E. A. Shaffer. This is an open access article distributed under the Creative Commons Attribution License, which permits unrestricted use, distribution, and reproduction in any medium, provided the original work is properly cited.

\begin{abstract}
Pneumatosis intestinalis is a rare disorder characterized by gas-filled cysts within the subserosal and/or submucosal regions of the intestinal wall. The source of this gas and its translocation across the mucosa is incompletely understood. Most (85\%) cases are associated with medical conditions, ranging from psychiatric through respiratory disorders to gastrointestinal-related diseases; the remaining 15\% lack any recognizable cause or association. In this case report, pneumatosis coli (affecting the colon) occurred in a patient following abdominal surgery and was associated with pseudomembranous colitis, which was Clostridium difficile toxin negative-presumably a false negative. Supportive care and appropriate antibacterial agents sufficed to alleviate symptoms and resolve the pneumatosis. Recognizing this uncommon but important association can avoid high financial and personal costs from unnecessary testing and invasive surgical explorations. Consideration should be given to pseudomembranous colitis as the basis for pneumatosis coli developing in patients who have received antibiotics, once gut ischemia has been ruled out.
\end{abstract}

\section{Introduction}

Pneumatosis intestinalis (PI) is characterized by multiple, thin-walled, gas-filled cysts within the subserosal and submucosal regions of the intestinal wall. Typically the location of pneumatosis is $46 \%$ in the colon, $27 \%$ small bowel, $5 \%$ stomach (usually termed gastric pneumatosis), and $7 \%$ involving both the small and large intestine [1]. Its pathogenesis is not completely understood. $15 \%$ of cases are primary or idiopathic, without identifiable cause or association. $85 \%$ of cases are associated with a wide variety of medical conditions and infections, suggesting a number of potential underlying pathogenic processes that may contribute to its development, that is, as a secondary event [2]. When limited to the colon, such collections of intramural gas are termed pneumatosis coli (PC).

We report a case of PC in a patient following colonic surgery, which was diagnosed as pseudomembranous colitis and successfully treated for a toxin-negative Clostridium difficile infection.

\section{Case Presentation}

A 56-year-old male with recently diagnosed rectal carcinoma (T1N0M0) underwent a low anterior resection and diversion ileostomy that was followed four weeks later by an uncomplicated loop ileostomy closure. Within a week of this reversal surgery, he was readmitted with nausea, vomiting, anorexia, and diffuse abdominal pain, along with 6-8 nonbloody, small volume, liquid bowel movements daily. The patient had not been taking proton pump inhibitors, but had received ceftazidime and gentamicin with his recent surgeries. He appeared unwell on admission with nonspecific abdominal tenderness upon palpation; bowel sounds were normal. His hemoglobin was $130 \mathrm{~g} / \mathrm{dL}$, platelet count $416 \times 10^{9} / \mathrm{L}$, and white blood cell count $6.7 \times 10^{9} / \mathrm{L}$. Electrolytes, including bicarbonate, and creatinine were also within normal limits. The initial stool collections, were negative for the $C$. difficile cytotoxin assay (C. difficile Tox A/B II (Techlab, Blacksburg, VA)), bacterial culture, and ova/parasites. Abdominal X-rays revealed distended loops of large bowel with mural edema. 


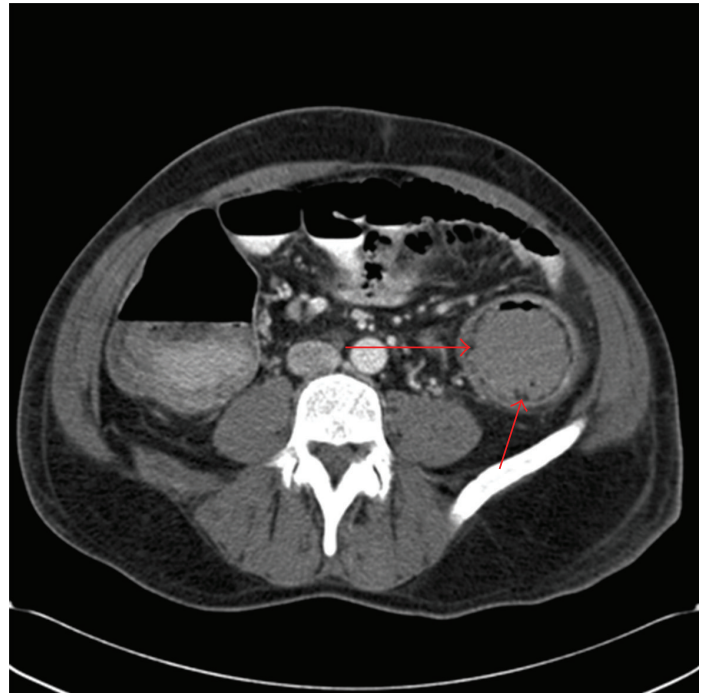

Figure 1: Abdominal computed tomography (CT) revealing submucosal air-fluid cysts. Arrows demarcate submucosal gas within the wall of the left colon, pneumatosis coli.

Subsequent abdominal computerized tomography (CT) demonstrated extensive pneumatosis coli (Figure 1) involving the descending colon; there was also thumb printing and dilated loops of bowel. The patient was initially placed on metronidazole $500 \mathrm{mg}$ IV q8h for three days due to concern regarding the clinical suspicion for a Clostridium difficile associated diarrheal (CDAD) infection. The intravenous route was chosen because of the postsurgical ileus with abdominal distension, reduced bowel sounds, and inability to tolerate oral feeds. A mild metabolic alkalosis was present on blood gas but there was no evidence of acidosis that might have suggested a gut ischemia. Flexible sigmoidoscopy clinically confirmed the diagnosis, showing quite classical pseudomembranes in addition to features consistent with pneumatosis intestinalis (Figure 2). Biopsies revealed a focal active colitis with superficial ulceration, gland destruction, and areas in which exudates overlay the colonic mucosa, all supporting the diagnosis of pseudomembranous colitis. Two subsequent $C$. difficile toxin assays however proved negative. The intravenous metronidazole was then changed to oral vancomycin at $125 \mathrm{mg}$ four times daily for four days. The patient improved over the subsequent week with a reduction in the frequency of bowel motions and a marked improvement in abdominal discomfort. Follow-up abdominal CT scan 8 days after the initial study revealed resolution of the pneumatosis coli. Discharged thereafter, he remained well without a recurrence of either entity; his last colonoscopy was normal 7 months following discharge.

\section{Discussion}

3.1. Epidemiology. Pneumatosis intestinalis, rather than being a disease per se, better relates to the underlying pathological process that varies widely: from being quite incidental without symptoms detected on screening colonoscopy to

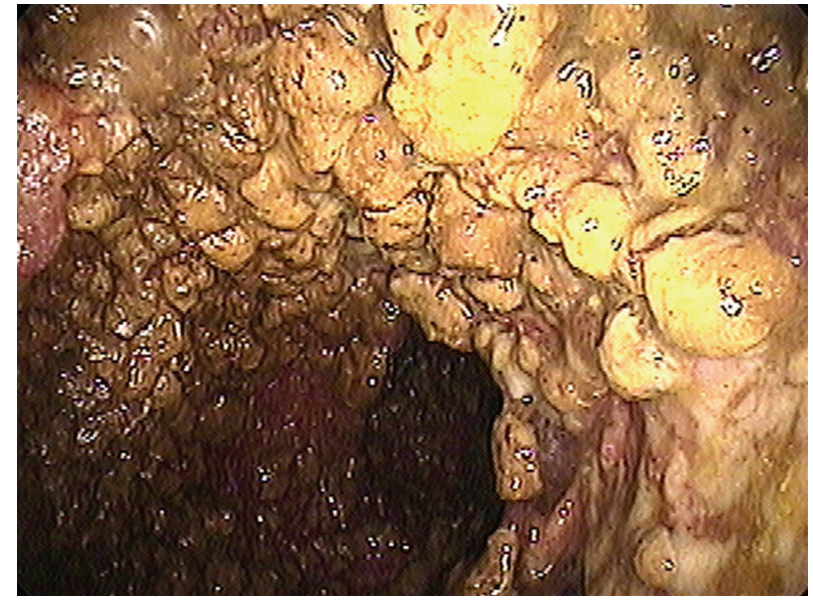

Figure 2: Pseudomembranes and pneumatosis coli. Endoscopic evaluation of the colonic mucosa reveals pseudomembranes. Cystic lesions are evident beneath the pseudomembranous layer.

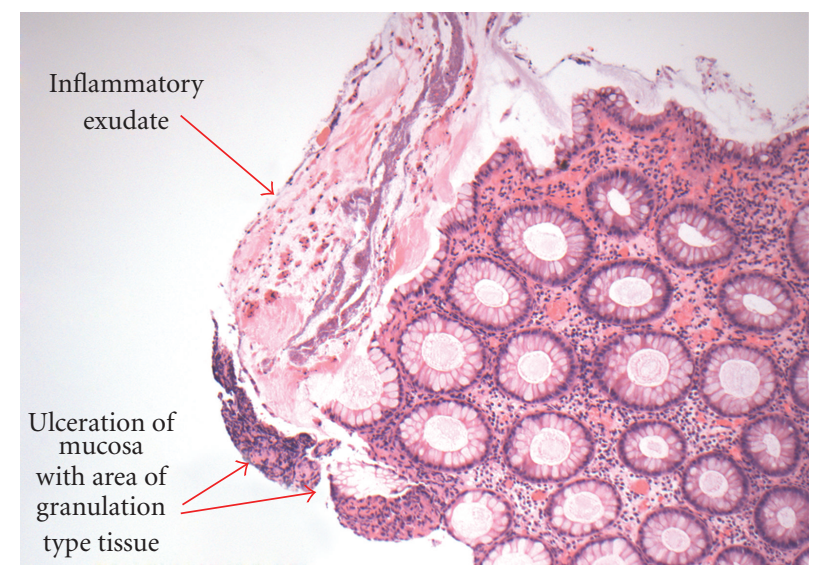

FIgUre 3: Colonic biopsy (10x) showing changes with exudate and ulceration (arrows) that are typical of the pseudomembrane formation seen in pseudomembranous colitis.

being associated with life-threatening conditions, such as necrotizing enterocolitis in infants or ischemic/infracted bowel in adults [3]. Though a rare event, pneumatosis has historically mandated surgical exploration and was associated with a high mortality rate $(33 \%)$, mostly reported as small case series [1]. A more recent study that reviewed 25,000 CT scans done over a 7-year period identified a prevalence of PI in $0.37 \%$ of patients [1]. Approximately $50 \%$ of these patients however were successfully managed nonoperatively. As many cases of pneumatosis are incidental findings detected on diagnostic imaging, its true frequency must be underestimated.

3.2. Clinical Associations. PI in adults is typically divided into primary ( $15 \%$ of cases being idiopathic, lacking any identifiable association) and secondary (85\%) forms with diverse clinical associations, including psychiatric disorders, 
respiratory conditions, dementia, inflammatory bowel disease, diverticular disease, redundant sigmoid colon, appendicitis, peptic ulcer disease, and within the past decade, bacterial infections like $C$. difficile [4-6]. PI affecting infants is life-threatening, occurring in the setting of necrotizing enterocolitis as well as PC that usually presents as a milder form of this entity [7].

3.3. Pathophysiology. These multiple, gas-filled cysts (actually pseudocysts: spaces without a distinctive epithelial membrane) develop in the intestinal submucosa and subserosa, presumably by air or gas translocating into the bowel wall $[8,9]$. A mechanical theory raises the concept of mucosal disruption through direct trauma or increased intraluminal pressure, permitting intraluminal gas to dissect directly into the submucosa or track via mesenteric vessels into the subserosa [8]. Such mucosal disruption could result from an endoscopic procedure, a perforated ulcer, inflammatory bowel disease, necrotizing enterocolitis, intestinal ischemia, diverticulosis, and after intestinal anastamosis, all associated with the development of pneumatosis. Nevertheless, there has been no documented anatomic connection between the mucosa and the cysts. A pulmonic theory invokes rupture of a pulmonary bleb though increased intrathoracic pressures (e.g., coughing), allowing air to transect through the mediastinum into the retroperitoneum, the perivascular spaces and thence the subserosa. Unexplained are the paucity of reports showing mediastinal air on diagnostic imaging [10]. Furthermore, a bacterial theory suggests that luminal bacteria enhance gas production (e.g., hydrogen gas from fermented carbohydrates) and this endogenous gas then diffuses across the mucosa [8]. In animal models, introduction of gas-forming bacteria can induce PC [11-14], which resolves with subsequent antibiotic therapy $[12,13,15]$. This mechanism however would presumably only explain PC, as opposed to PI, because hydrogen-producing bacteria reside in the colon and not the small bowel.

3.4. Clinical Presentation. The clinical picture can be quite varied depending on the underlying etiology and if any complications have transpired relative to the pneumatosis. The spectrum of pneumatosis nevertheless does include abdominal sepsis and death, often associated with evidence of portal venous gas $[1,2,16]$. Most commonly, patients have diarrhea, mucus discharge, rectal bleeding, and constipation [6]. Complications associated with PC include pneumoperitoneum, volvulus, intestinal obstruction, perforation and intussusception. In infants, pneumatosis intestinalis that develops secondarily to necrotizing enterocolitis culminates in a high mortality rate.

3.5. Diagnosis. Investigations should start with plain abdominal X-rays that may reveal complications related to PC; these are present in $23 \%$ [1]. Such findings include radiolucent clusters, linear streaks, or small bubbles along the bowel wall. If a cyst ruptures, a pneumoperitoneum develops. Barium enema might demonstrate the gas-filled cysts in the wall of the colon, but is not routinely ordered. Instead, CT imaging typically follows the abdominal X-ray in clinical practice. CT accurately identifies the bubble-like collections of gas within the submucosal and subserosal layers of the colonic mucosa. Evidence of pneumatosis when combined with prominent portomesenteric venous gas represents ominous findings, suggesting transmural bowel infarction. These impressive radiologic features however are not absolute prognostic indicators as CT imaging detects pneumatosis at a relatively early stage that may only signify partial mural bowel ischemia [16]. Care must be taken to also differentiate bubble-like PI from pneumatosis linearis: bands of gas within the bowel wall that place here associated with loss of gut viability, prompting urgent surgery. Ultimately an endoscopic evaluation may become necessary to differentiate PC from familial adenomatous polyposis and colonic malignancies. Intestinal biopsies in PI characteristically reveal gas-filled spaces within the submucosa that on histopathology are lined with histiocytes and multinucleated giant cells when involving the subserosa.

3.6. Diagnosis of Clostridium Difficile Infection. Key in managing PC is to identify a correctable etiology. In the present case, suspicion of a Clostridium difficile infection (CDI) arose because watery diarrhea had developed in a recently hospitalized patient who had received antibacterial agents $[17,18]$. (Watery diarrhea in this context has been defined as $\geq 3$ stools per day that are sufficiently liquid to conform to the shape of its laboratory container $[16,17])$. The diagnosis of pseudomembranous colitis however came from diagnostic imaging, the endoscopy showing pseudomembranes, and the characteristic histopathology findings. Our patient had $C$. difficile toxin assays that were negative on three separate occasions.

C. difficile is a gram positive, rod-shaped bacterium. CDI results from pathogenic strains that are capable of producing two structurally similar toxins: A and B. Both are important in producing $C$. difficile colitis: toxin A being considered enterotoxigenic whereas toxin $B$ has potent cytotoxic effects [19]. Hence, the laboratory diagnosis comes in two formats: (1) Toxin assays for one or both toxins, or for the cytotoxic effect, and (2) Microorganism assays to test for a common antigen or perform an anaerobic stool culture for C. difficile. Commercially available enzyme immunoassays (EIAs) are capable of detecting both toxin A and toxin B, important as occasionally some strains produce only A or only B. [20]. Although EIAs are rapid, inexpensive, and yield high specificity (up to 99\%), their rather modest sensitivity (32$73 \%$ ) results in a high false negative rate [18]. Repeating the stool EIA, as was done here when the initial test was negative, may improve sensitivity but only slightly $(\sim 10 \%)$. Re-ordering such stool tests moreover ultimately reduces specificity to unacceptable levels $[17,18]$. More sensitive polymer chain reaction (PCR) assays for the toxin genes, recent additions, provide high sensitivity in the order of $97 \%$. Lastly, tissue culture to detect cell cytotoxicity is costly and impractical despite being a "gold" standard for diagnosing C. difficile.

The second category checks for the presence of the organism. Common antigen testing seeks to detect the enzyme, glutamate dehydrogenase $(\mathrm{GDH})$, that $C$. difficile 
produces [21]. EIA assays for GDH carry a high sensitivity (85-95\%) while ensuring a high negative predictive value [21]. The GDH assay (like the cumbersome anaerobic stool culture) unfortunately also detects non-toxigenic and hence clinically irrelevant isolates. A strategy suggested by the 2010 IDSA guidelines on $C$. difficile supports a two step method: EIA to detect GDH as the initial screen (which is most sensitive), followed by a cell cytotoxicity assay, toxigenic culture or PCR for the toxin gene as the confirmatory test for GDHpositive specimens only, hence eliminating false-positive GDH results [21]. Culturing for $C$. difficile may be sensitive (over 90\%), but is not practical as a routine diagnostic test since the results require 48-72 hours to complete. Further, anaerobic stool culture does not differentiate between toxinproducing and non-toxigenic strains. Culture is best reserved for epidemiologic, infection control or antibacterial studies.

As no test combination to date is $100 \%$ sensitive and specific, endoscopy is an important diagnostic tool. Morphological diagnosis, as was necessary in this difficult case, came from the endoscopic picture and biopsy. Nevertheless, pseudomembranes are only detected endoscopically in about $50 \%$ of CDAD cases, as confirmed by both stool culture and cytotoxin stool test positivity [22]. Conversely, the presence of pseudomembranes must be taken in the clinical context, being neither absolutely sensitive nor specific for $C$. difficile colitis; pseudomembranes can accompany ischemic colitis and inflammatory bowel disease.

Some endoscopic similarities exist between ischemic colitis and CDI-associated pseudomembranous colitis, further complicating such difficult acute abdominal diagnoses. In ischemic colitis, however, any pseudomembranes tend to be more diffuse and have transmural involvement, compared to $C$. difficile colitis. Further, the histological presence of atrophic microcrypts, lamina propria hemorrhage, fullthickness necrosis and hyalinized lamina propria are specific markers for ischemia [23].

The diagnosis of pseudomembranous colitis in the current case is secure from the endoscopic and morphological perspectives. The presence of pseudomembranous colitis is strongly suggestive of toxigenic $C$. difficile infection regardless of the results of toxin EIA despite the absence of backup tests, such as cytotoxicity, culture, or PCR. Therefore, one of two conclusions is possible. (1) This was a toxigenic infection with a false-negative EIA-most likely, or (2) this was an extremely rare case of pseudomembranous colitis due to a cause other than C. difficile.

3.7. Prognosis and Management. The course of pneumatosis is quite variable, largely determined by the underlying disease process, especially those leading to bowel infraction or perforation. Traditionally, a diagnosis of PI was associated with a high mortality rate and mandated aggressive surgical intervention. In fact, the clinical course is generally quite benign: most $(\sim 50 \%)$ cases of PI spontaneously resolve, particularly when idiopathic. Regardless of PI location or intervention, there remains a significant mortality at $20 \%-$ $40 \%$, emphasizing the need to differentiate between sinister gut ischemia and rather benign PI [1]. Hence, interventions for imaging-diagnosed PI generally may be categorized as (a) operative (about 1/3rd), (b) nonoperative $(\sim 1 / 2)$, and (c) futile $(\sim 15 \%)$, favoring non-surgical management such as inserting a nasogastric tube supported by IV fluids.

The key decision therefore is whether to perform an emergency laparotomy for an acute intra-abdominal catastrophe or to manage the case conservatively for rather benign PI. A quite reasonable guide to identify gut infarction and the need for surgical intervention is a clinical setting that suggests an acute abdomen, laboratory evidence of a metabolic acidosis (particularly lactic acidosis) and elevated amylase, and diagnostic imaging showing linear pneumatosis and/or portal venous gas [24].

Specific treatment is best directed at the underlying disease process. For those with symptoms, management strategies directed at the pneumatosis itself have used (1) Oxygen therapy (either by inhalation or as hyperbaric therapy, the latter to reduce toxicity) for several days to impede anaerobic bacteria that produce the hydrogen and other gases in these cysts, and to replace these gases [11]; (2) antibacterial agents (like metronidazole) 7 days for up to 2-3 months to decrease bacterial gas production [12, 25]; (3) elemental diet for 2 weeks, seeking to alter the colonic microflora [26]. Colonic resection is occasionally necessary for complications like bowel obstruction. For the majority without symptoms, an expectant approach is all that is necessary because the intramural gas should resolve with time.

\section{Acknowledgments}

The authors appreciate the histopathological expertise provided by Dr. Anna Sienko, Department of Pathology, the advice offered by Dr. Shane Devlin in preparing this paper, and the valued microbiological information supplied by Drs. Dan Gregson and Thomas Louis, Division of Medical Microbiology, Departments of Medicine and Pathology \& Laboratory Medicine, University of Calgary, Calgary, Alberta.

\section{References}

[1] M. S. Morris, A. C. Gee, S. D. Cho et al., "Management and outcome of pneumatosis intestinalis," American Journal of Surgery, vol. 195, no. 5, pp. 679-683, 2008.

[2] L. G. Koss, "Abdominal gas cysts (pneumatosis cystoides intestinorum hominis); an analysis with a report of a case and a critical review of the literature," American Architect Pathology, vol. 53, no. 6, pp. 523-549, 1952.

[3] Y. Heng, M. D. Schuffler, R. C. Haggitt, and C. A. Rohrmann, "Pneumatosis intestinalis: a review," American Journal of Gastroenterology, vol. 90, no. 10, pp. 1747-1758, 1995.

[4] C. Kreiss, F. Forohar, A. E. Smithline, and L. J. Brandt, "Pneumatosis intestinalis complicating C. difficile pseudomembranous colitis," American Journal of Gastroenterology, vol. 94, no. 9, pp. 2560-2561, 1999.

[5] P. Schenk, C. Madl, L. Kramer et al., "Pneumatosis intestinalis with Clostridium difficile colitis as a cause of acute abdomen after lung transplantation," Digestive Diseases and Sciences, vol. 43, no. 11, pp. 2455-2458, 1998. 
[6] G. Gagliardi, I. W. Thompson, M. J. Hershman, A. Forbes, P. R. Hawley, and I. C. Talbot, "Pneumatosis coli: a proposed pathogenesis based on study of 25 cases and review of the literature," International Journal of Colorectal Disease, vol. 11, no. 3, pp. 111-118, 1996.

[7] J. C. Leonidas and R. T. Hall, "Neonatal pneumatosis coli: a mild form of neonatal necrotizing enterocolitis," Journal of Pediatrics, vol. 89, no. 3, pp. 456-459, 1976.

[8] S. U. Christl, G. R. Gibson, P. R. Murgatroyd, W. Scheppach, and J. H. Cummings, "Impaired hydrogen metabolism in pneumatosis cystoides intestinalis," Gastroenterology, vol. 104, no. 2, pp. 392-397, 1993.

[9] M. D. Levitt and S. Olsson, "Pneumatosis cystoides intestinalis and high breath $\mathrm{H} 2$ excretion: insights into the role of $\mathrm{H} 2$ in this condition," Gastroenterology, vol. 108, no. 5, pp. 1560$1565,1995$.

[10] N. Gullà, V. Castagna, V. Dei Santi et al., "Pneumoretroperitoneum caused by pneumatosis cystoides intestinalis. Etiopathogenethical clinical and diagnostic considerations. Report of a clinical case," Minerva Chirurgica, vol. 53, no. 9, pp. 757-765, 1998.

[11] H. H. Stone, W. B. Allen, R. B. Smith III, and C. D. Haynes, "Infantile pneumatosis intestinalis," Journal of Surgical Research, vol. 8, no. 7, pp. 301-307, 1968.

[12] S. Holt, H. M. Gilmour, and T. A. S. Buist, "High flow oxygen therapy for pneumatosis coli," Gut, vol. 20, no. 6, pp. 493-498, 1979.

[13] J. Wandtke, J. Skucas, R. Spataro, and R. J. Bruneau, "Pneumatosis intestinalis as a complication of jejunoileal bypass," American Journal of Roentgenology, vol. 129, no. 4, pp. 601604, 1977.

[14] S. D. St. Peter, M. A. Abbas, and K. A. Kelly, "The spectrum of pneumatosis intestinalis," Archives of Surgery, vol. 138, no. 1, pp. 68-75, 2003.

[15] A. Al-Talib, F. Al-Ghtani, and R. Munk, "Pneumatosis Intestinalis: can we avoid surgical intervention in nonsurgical patients?" Case Reports in Gastroenterology, vol. 3, pp. 286292, 2009.

[16] W. Wiesner, K. J. Mortelé, J. N. Glickman, H. Ji, and P. R. Ros, "Pneumatosis intestinalis and portomesenteric venous gas in intestinal ischemia: correlation of CT findings with severity of ischemia and clinical outcome," American Journal of Roentgenology, vol. 177, no. 6, pp. 1319-1323, 2001.

[17] T. Planche, A. Aghaizu, R. Holliman et al., "Diagnosis of Clostridium difficile infection by toxin detection kits: a systematic review," The Lancet Infectious Diseases, vol. 8, no. 12, pp. 777-784, 2008.

[18] L. R. Peterson and A. Robicsek, "Does my patient have Clostridium difficile infection?" Annals of Internal Medicine, vol. 151, no. 3, pp. 176-179, 2009.

[19] D. Lyras, J. R. O'Connor, P. M. Howarth et al., "Toxin B is essential for virulence of Clostridium difficile," Nature, vol. 458, no. 7242, pp. 1176-1179, 2009.

[20] D. Drudy, S. Fanning, and L. Kyne, "Toxin A-negative, toxin B-positive Clostridium difficile," International Journal of Infectious Diseases, vol. 11, no. 1, pp. 5-10, 2007.

[21] S. H. Cohen, D. N. Gerding, S. Johnson et al., "Clinical practic guidelines for clostridium difficile infection in adults: 2010 update by the society for healthcare epidemiology of america (SHEA) and the infectious diseases society of america (IDSA)," Infection Control Hospital Epidemiology, vol. 31, no. 5, pp. 431-455, 2010.
[22] D. N. Gerding, M. M. Olson, and L. R. Peterson, "Clostridium difficile-associated diarrhea and colitis in adults. A prospective case-controlled epidemiologic study," Archives of Internal Medicine, vol. 146, no. 1, pp. 95-100, 1986.

[23] C. R. Dignan and J. K. Greenson, "Can ischemic colitis be differentiated from C difficile colitis in biopsy specimens?" American Journal of Surgical Pathology, vol. 21, no. 6, pp. 706710, 1997.

[24] S. J. Knechtle, A. M. Davidoff, and R. P. Rice, "Pneumatosis intestinalis. Surgical management and clinical outcome," Annals of Surgery, vol. 212, no. 2, pp. 160-165, 1990.

[25] N. Ade-Ajayi, P. Veys, M. Stanton, D. P. Drake, and A. Pierro, "Conservative management of pneumatosis intestinalis and pneumoperitoneum following bone-marrow transplantation," Pediatric Surgery International, vol. 18, no. 8, pp. 692695, 2002.

[26] B. T. Johnston and R. J. McFarland, "Elemental diet in the treatment of pneumatosis coli," Scandinavian Journal of Gastroenterology, vol. 30, no. 12, pp. 1224-1227, 1995. 


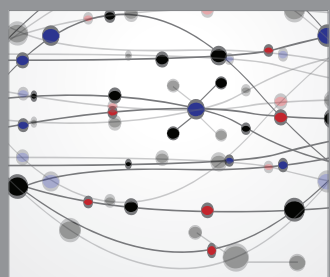

The Scientific World Journal
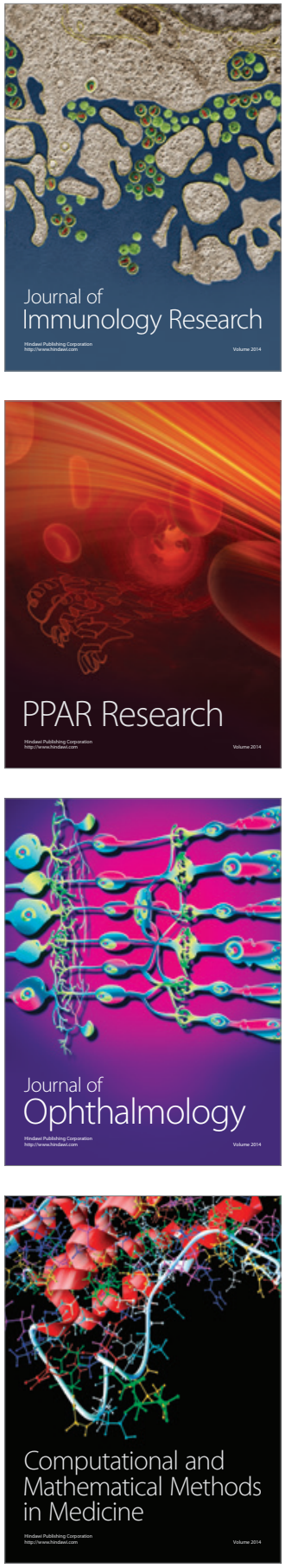

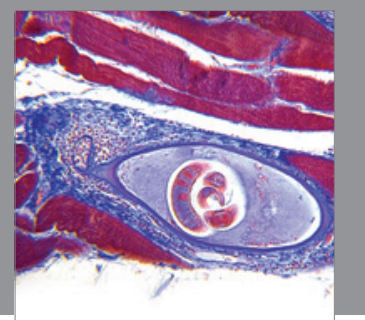

Gastroenterology

Research and Practice
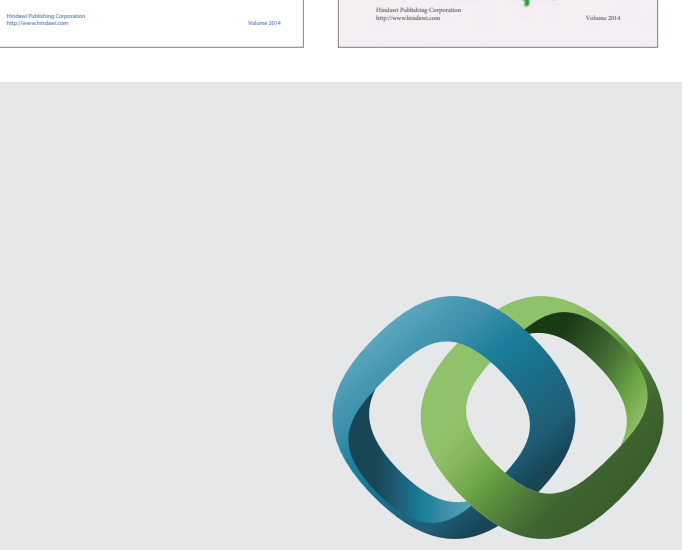

\section{Hindawi}

Submit your manuscripts at

http://www.hindawi.com
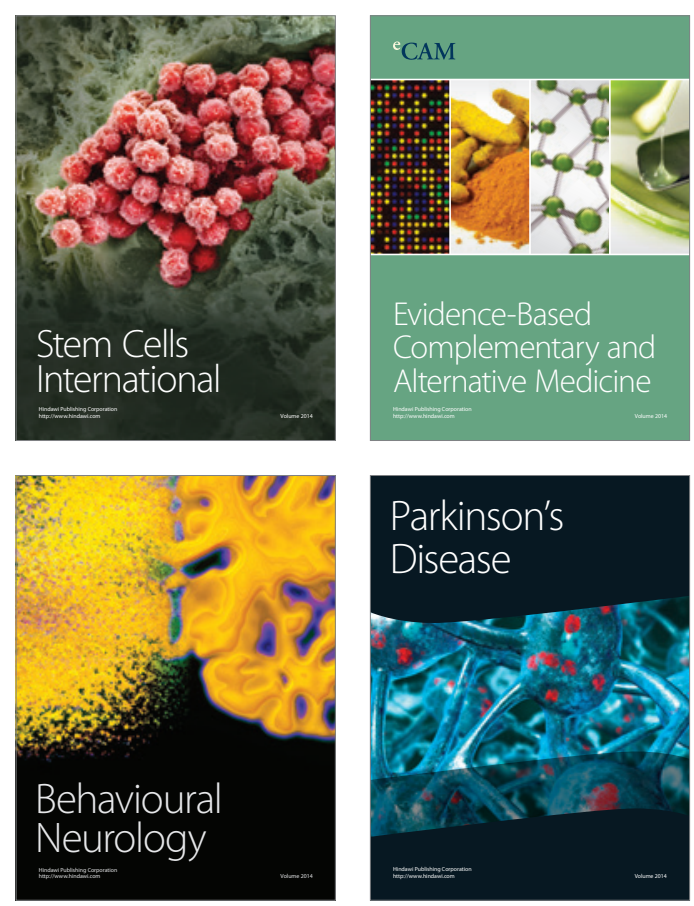

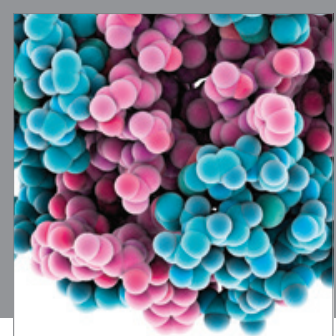

Journal of
Diabetes Research

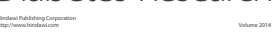

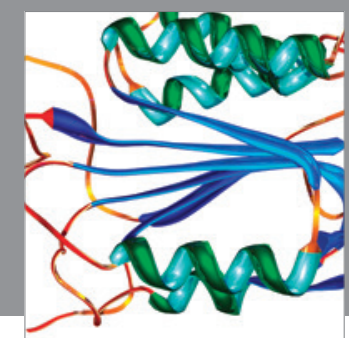

Disease Markers
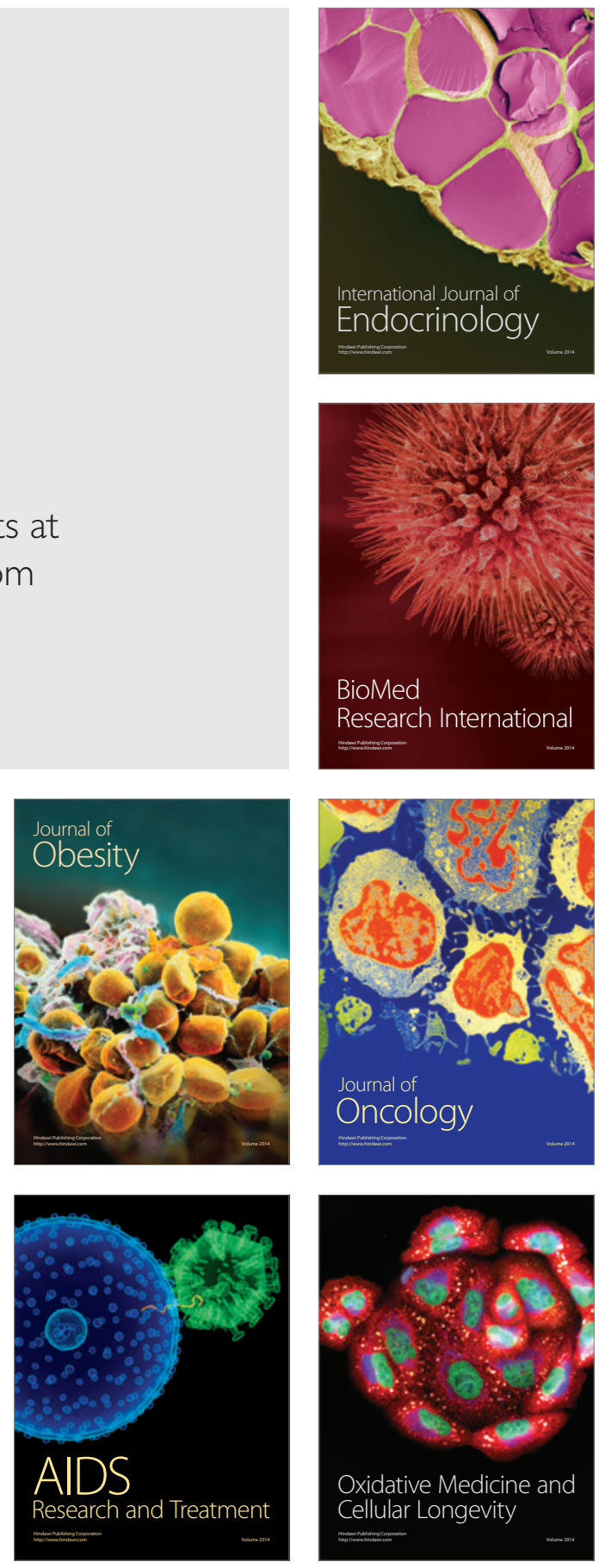